\title{
GB Virus C E2 Inhibits PD-1-Mediated T Cell Signaling Dysfunction during Chronic Viral Infection ${ }^{\dagger}$
}

\author{
Nirjal Bhattarai 1,2,3, Jennifer L. Welch 1,2, Jinhua Xiang 1,2, Muthu Saravanan Manoharan 4,5, \\ Jeffrey A. Martinson ${ }^{6}$, Alan L. Landay ${ }^{6}$, Sunil K. Ahuja a,5, James H. McLinden ${ }^{1,2}$ \\ and Jack T. Stapleton $1,2,7, *$ \\ 1 Department of Internal Medicine, University of Iowa, Iowa City, IA 52242, USA; \\ Nirjal.Bhattarai@fda.hhs.gov (N.B.); jennifer-welch@uiowa.edu (J.L.W.); jinhua-xiang@uiowa.edu (J.X.); \\ james-mclinden@uiowa.edu (J.H.M.) \\ 2 Iowa City VA Health Care System, Iowa City, IA 52242, USA \\ 3 Division of Cellular and Gene Therapies, Center for Biologics Evaluation and Research, \\ U.S. Food and Drug Administration, Silver Spring, MD 20993, USA \\ 4 Veterans Administration Center for Personalized Medicine, South Texas VA Health Care System, \\ San Antonio, TX 78229, USA; Manoharan@uthscsa.edu (M.S.M.); AHUJAS@uthscsa.edu (S.K.A.) \\ 5 Department of Medicine, University of Texas Health Science Center, San Antonio, TX 78229, USA \\ 6 Department of Immunology-Microbiology, Rush University Medical Centre, Chicago, IL 60612, USA; \\ jmartins@rush.edu (J.A.M.); alanday@rush.edu (A.L.L.) \\ 7 Department of Microbiology \& Immunology, University of Iowa, Iowa City, IA 52242, USA \\ * Correspondence: jack-stapleton@uiowa.edu \\ † Presented at Viruses 2020-Novel Concepts in Virology, Barcelona, Spain, 5-7 February 2020. \\ Published: 16 June 2020
}

\begin{abstract}
Background: Program death receptor 1 (PD-1) is a co-inhibitory receptor that is upregulated and contributes to $\mathrm{T}$ cell dysfunction (exhaustion) during chronic viral infections, including HIV and $\mathrm{HCV}$. GB virus C (GBV-C) is a persistent human virus, and co-infection is associated with reduced immune activation and improved clinical outcomes in HIV- and Ebola-infected individuals. Methods: PD-1 levels were measured by flow cytometry on CD38+ T cells from 45 HIV-infected individuals, 20 of whom were co-infected with GBV-C. Jurkat cell lines that stably express GBV-C E2 protein and vector control were used to purify total cellular RNA before, and $24 \mathrm{~h}$ following, activation using anti-CD3/CD28 treatment. Gene expression was analyzed by RNA-seq and qRT-PCR. Results: HIV-infected individuals with GBV-C viremia had reduced PD-1 expression on activated CD4+ and CD8+ T cells compared to HIV-infected GBV-C negative individuals. GBV-C particles and GBV-C E2 protein each inhibited PD-1 expression on T cells in vitro. Consistent with this, GBV-C E2 reduced gene expression of $P D-1$, and its ligand $P D-L 1$, in both resting and activated $T$ cells. GBV-C E2 regulated transcription of the PD-1 signaling pathway and T cell activation associated genes, without downregulation of the interferon-stimulated and innate immunity-related genes needed to resolve viral infections. Conclusions: Our current understanding of chronic RNA virus infections is that upregulation of PD-1 with $\mathrm{T}$ cell exhaustion is critical for viral persistence. However, these data demonstrate that GBV-C infection reduced PD-1 expression on activated T cells during HIV infection, and that the GBV-C E2 protein inhibits PD-1 signaling in T cells. This may preserve $\mathrm{T}$ cell function and contribute to the lack of immune deficiency in people with chronic GBV-C infection. Understanding the mechanisms by which GBV-C E2 alters PD-1 signaling may aid in the development of novel immunomodulatory therapeutics to prevent $\mathrm{T}$ cell dysfunction (exhaustion) during chronic viral infections.
\end{abstract}


Keywords: GBV-C; PD-1; T cell exhaustion; HIV

(c)

(C) 2020 by the authors. Licensee MDPI, Basel, Switzerland. This article is an open access article distributed under the terms and conditions of the Creative Commons Attribution (CC BY) license (http://creativecommons.org/licenses/by/4.0/). 